\title{
Numerical Integration Formulas of Degree Two
}

\author{
By A. H. Stroud
}

1. Introduction. Here we discuss numerical integration formulas of the form

$$
\int_{R} f(x) w(x) d x \cong \sum_{i} a_{i} f\left(\nu_{i}\right)
$$

where $R$ is a region in $n$-dimensional, real, euclidean space; $x=\left(x_{1}, x_{2}, \cdots, x_{n}\right)$; the $a_{i}$ are constants; and the $\nu_{i}$ are points in the space. Most previous authors have given formulas for special regions (for a bibliography see [4]). Thacher [7] has given a method for constructing formulas of degree 2 with $n+1$ points for general regions and of degree 3 with $2 n$ points for certain symmetric regions; with his method, however, each region must also be treated separately. Our main results are to obtain specific formulas of degree 2 with $n+1$ points for a general region satisfying a certain condition of non-degeneracy, and to show that for these regions such formulas cannot be obtained with fewer points. We also give a specific $2 n$ point formula of degree 3 for a general centrally symmetric region. These results are a generalization of those of Georgiev $[1,2,3]$ who has obtained similar results (but gives no general formulas) for $n=2,3$ with $w(x) \equiv 1$. Our results are obtained by a different method which was developed without knowledge of Georgiev's work.

2. Formulas of degree 2. We assume at first that an integration formula of degree 2 for $R$ with respect to $w(x)$ can be obtained with $n+1$ points

$$
\nu_{i}=\left(\nu_{i 1}, \cdots, \nu_{i n}\right), \quad i=0,1, \cdots, n \text {. }
$$

Then the equations

$$
\begin{aligned}
& a_{0}+a_{1}+\cdots+a_{n}=c_{0} \\
& a_{0} \nu_{0 j}+a_{1} \nu_{1 j}+\cdots+a_{n} \nu_{n j}=c_{0 j} \\
& a_{0} \nu_{0 j} \nu_{0 k}+a_{1} \nu_{1 j} \nu_{1 k}+\cdots+a_{n} \nu_{n j} \nu_{n k}=c_{j k} \quad j, k=1,2, \cdots, n
\end{aligned}
$$

must be solved for both the $a_{i}$ and the $\nu_{i}$, where

$$
c_{0}=\int_{R} w(x) d x, \quad c_{0 j}=\int_{R} x_{j} w(x) d x, \quad c_{j k}=\int_{R} x_{j} x_{k} w(x) d x .
$$

We begin by writing (1) as the matrix equation

$$
U^{\top} A U=C
$$

where

$U=\left[\begin{array}{cccc}1 & \nu_{01} & \cdots & \nu_{0 n} \\ 1 & \nu_{11} & \cdots & \nu_{1 n} \\ & & \cdots & \\ 1 & \nu_{n 1} & \cdots & \nu_{n n}\end{array}\right] \quad A=\left[\begin{array}{cccc}a_{0} & 0 & \cdots & 0 \\ 0 & a_{1} & \cdots & 0 \\ & & \cdots & \\ 0 & 0 & \cdots & a_{n}\end{array}\right] \quad C=\left[\begin{array}{cccc}c_{0} & c_{01} & \cdots & c_{0 n} \\ c_{01} & c_{11} & \cdots & c_{1 n} \\ & \cdots & & \\ c_{0 n} & c_{1 n} & \cdots & c_{n n}\end{array}\right]$ and where we assume $0<c_{0}<\infty$ and $0<|\operatorname{det} C|<\infty$.

Received March 18, 1958; in revised form August 4, 1959. This work was supported in part by the Office of Ordnance Research, U. S. Army and in part by the Wisconsin Alumni Research Foundation. 
Since $C$ is non-singular we can find a matrix $T$ such that

$$
T^{\top} U^{\top} A U T=T^{\top} C T=c_{0} E
$$

where $E$ is a diagonal matrix with elements \pm 1 . The method for finding $T$ is well known (see [5], p. 56); we illustrate it using $n=3$.

Since $c_{0} \neq 0$ we define $t_{0 i}=-c_{0 i} / c_{0}, i=1,2,3$, and form

$$
T_{1}=\left[\begin{array}{cccc}
1 & t_{01} & t_{02} & t_{03} \\
0 & 1 & 0 & 0 \\
0 & 0 & 1 & 0 \\
0 & 0 & 0 & 1
\end{array}\right]
$$

$$
C_{1}=T_{1}^{\top} C T_{1}=\left[\begin{array}{cccc}
c_{0} & 0 & 0 & 0 \\
0 & c_{11}^{(1)} & c_{12}^{(1)} & c_{13}^{(1)} \\
0 & c_{12}^{(1)} & c_{22}^{(1)} & c_{23}^{(1)} \\
0 & c_{13}^{(1)} & c_{23}^{(1)} & c_{23}^{(1)}
\end{array}\right]
$$

Now if $c_{11}^{(1) *}=0$ some $c_{1 i}^{(1)} \neq 0$ since $\operatorname{det} C \neq 0$. Assuming $c_{12}^{(1)} \neq 0$ we form

$$
T_{2}=\left[\begin{array}{llll}
1 & 0 & 0 & 0 \\
0 & 1 & 0 & 0 \\
0 & h & 1 & 0 \\
0 & 0 & 0 & 1
\end{array}\right] \quad C_{2}=T_{2}{ }^{\top} C_{1} T_{2}=\left[\begin{array}{cccc}
c_{0} & 0 & 0 & 0 \\
0 & 2 h c_{12}^{(1)}+h^{2} c_{22}^{(1)} & c_{12}^{(1)}+h c_{22}^{(1)} & c_{13}^{(1)}+h c_{23}^{(1)} \\
0 & c_{12}^{(1)}+h c_{22}^{(1)} & c_{22}^{(1)} & c_{23}^{(1)} \\
0 & c_{13}^{(1)}+h c_{23}^{(1)} & c_{23}^{(1)} & c_{33}^{(1)}
\end{array}\right]
$$

and choose $h$ so that $c_{11}^{(1)}=2 h c_{12}^{(1)}+h^{2} c_{22}^{(1)} \neq 0$; if $c_{11}^{(1) *} \neq 0$ we take $h=0$ so that $c_{11}^{(1)}=c_{11}^{(1) *}$. In this way we are assured that the element in the 1,1 position is $\neq 0$.

Similarly we may find matrices $T_{3}, T_{4}$ and $T_{5}$ such that

$$
C_{3}=T_{4}{ }^{\top} T_{3}{ }^{\top} C_{2} T_{3} T_{4}=\left[\begin{array}{cccc}
c_{0} & 0 & 0 & 0 \\
0 & c_{11}^{(1)} & 0 & 0 \\
0 & 0 & c_{22}^{(2)} & c_{23}^{(2)} \\
0 & 0 & c_{22}^{(2)} & c_{33}^{(2)}
\end{array}\right] \quad T_{6}{ }^{\top} C_{3} T_{3}=\left[\begin{array}{cccc}
c_{0} & 0 & 0 & 0 \\
0 & c_{11}^{(1)} & 0 & 0 \\
0 & 0 & c_{22}^{(2)} & 0 \\
0 & 0 & 0 & c_{33}^{(3)}
\end{array}\right],
$$

where $c_{22}^{(2)}$ and $c_{33}^{(3)}$ are $\neq 0$. Defining $T_{6}$ as the diagonal matrix

$$
\left[1,\left[c_{0} /\left|c_{11}^{(1)}\right|\right]^{t},\left[c_{0} /\left|c_{22}^{(2)}\right|\right]^{\frac{1}{3}},\left[c_{0} /\left|c_{33}^{(3)}\right|\right]^{\frac{1}{1}}\right]
$$

we have finally $T=T_{1} T_{2} T_{3} T_{4} T_{3} T_{6}$.

We can assume $E$ has the form $[1,1, \ldots, 1,-1, \cdots,-1]$ since any other arrangement of +1 's and -1 's can be put into this form by a suitable interchange of the rows of $U T$ and the corresponding columns of $T^{\top} U^{\top}$. If $C$ is positive definite (for example if $w(x)$ is of constant sign on $R$ ) $E$ will be the identity. It should be noted that the first element of $E$ will always be positive.

In the following we write

$$
U T=\left[\begin{array}{cccc}
1 & \xi_{01} & \cdots & \xi_{0 n} \\
1 & \xi_{11} & \cdots & \xi_{1 n} \\
& & \cdots & \\
1 & \xi_{n 1} & \cdots & \xi_{n n}
\end{array}\right]=\left[\begin{array}{cccc}
1 & \nu_{01} & \cdots & \nu_{0 n} \\
1 & \nu_{11} & \cdots & \nu_{1 n} \\
& \cdots & \\
1 & \nu_{n 1} & \cdots & \nu_{n n}
\end{array}\right]\left[\begin{array}{cccc}
1 & \tau_{01} & \cdots & \tau_{0 n} \\
0 & \tau_{11} & \cdots & \tau_{1 n} \\
& \cdots & \\
0 & \tau_{n 1} & \cdots & \tau_{n n}
\end{array}\right] .
$$

Because $U T$ is non-singular and $E^{-1}=E$ we easily obtain from (3)

$$
(U T) E(U T)^{\top}=c_{0} A^{-1} \text {. }
$$


In terms of the $\xi_{i}$ this equation is

$$
\begin{aligned}
1+\xi_{i 1} \xi_{j 1}+\cdots+\xi_{i p} \xi_{j p}-\xi_{i, p+1} \xi_{j, p+1}-\cdots-\xi_{i n} \xi_{j n} & =\frac{c_{0}}{a_{1}} \delta_{i j} \\
& i, j,=0,1, \cdots, n .
\end{aligned}
$$

where $p+1,0 \leqq p \leqq n$, is the number of +1 's in $E$. We discuss the solution of (4); the $\nu_{i}$ are obtained from the $\xi_{i}$ by $\nu_{i j}=\tau_{0 j}^{\prime}+\xi_{i 1} \tau_{1 j}^{\prime}+\cdots+\xi_{i n}^{\prime} \tau_{n j}^{\prime}, i=$ $0,1, \cdots, n, j=1, \cdots, n$, where

$$
T^{-1}=\left[\begin{array}{cccc}
1 & \tau_{01}^{\prime} & \cdots & \tau_{0 n}^{\prime} \\
0 & \tau_{11}^{\prime} & \cdots & \tau_{1 n}^{\prime} \\
& & \cdots & \\
0 & \tau_{n 1}^{\prime} & \cdots & \tau_{n n}^{\prime}
\end{array}\right] .
$$

We are only interested in real solutions of (1) and therefore precisely $n-p+1$ of the $a_{i}$ must be negative by Sylvester's "law of inertia" ([5], p. 56). If $E$ is the identity $(p=n)$ clearly we must have $0<a_{i}<c_{0}$; if $p<n$ the only condition for the $a_{i}$ is that they be non-zero.

Table 1 gives a particular solution of (4); we have assumed $a_{0}, \cdots, a_{n-p}$ negative and $a_{n-p+1}, \cdots, a_{n}$ positive. In the places where a double sign occurs we mean to use the lower sign for the last $n-p$ components of each vector and the upper sign for the first $p$ components. Each $\xi_{i}$ is real.

TABLE 1

$$
\begin{aligned}
& \xi_{0}=\left(0,0, \cdots, 0,0,\left[\frac{c_{0}-a_{0}}{ \pm a_{0}}\right]^{1 / 2}\right) \\
& \xi_{1}=\left(0,0, \cdots, 0,\left[\frac{c_{0}\left(c_{0}-a_{0}-a_{1}\right)}{ \pm\left(c_{0}-a_{0}\right) a_{1}}\right]^{1 / 2}, \mp\left[\frac{ \pm a_{0}}{c_{0}-a_{0}}\right]^{1 / 2}\right) \\
& \xi_{2}=\left(0,0, \cdots,\left[\frac{c_{0}\left(c_{0}-a_{0}-a_{1}-a_{3}\right)}{ \pm\left(c_{0}-a_{0}-a_{1}\right) a_{2}}\right]^{1 / 2}, \mp\left[\frac{ \pm c_{0} a_{1}}{\left(c_{0}-a_{0}\right)\left(c_{0}-a_{0}-a_{1}\right)}\right]^{1 / 2}, \mp\left[\frac{ \pm a_{0}}{c_{0}-a_{0}}\right]^{1 / 2}\right) \\
& \xi_{n-2}=\left(0,\left[\frac{ \pm c_{0}\left(c_{0}-a_{0}-\cdots-a_{n-2}\right)}{\left(c_{0}-a_{0}-\cdots-a_{n-2}\right) a_{n-2}}\right]^{1 / 2}, \cdots\right. \\
& \left.\cdots, \mp\left[\frac{ \pm c_{0} a_{2}}{\left(c_{0}-a_{0}-a_{1}\right)\left(c_{0}-a_{0}-a_{1}-a_{2}\right)}\right]^{1 / 2},\left[\frac{ \pm c_{0} a_{1}}{\left(c_{0}-a_{0}\right)\left(c_{0}-a_{0}-a_{1}\right)}\right]^{1 / 2}, \mp\left[\frac{ \pm a_{0}}{c_{0}-a_{0}}\right]^{1 / 2}\right) \\
& \xi_{n-1}=\left(\left[\frac{ \pm c_{0}\left(c_{0}-a_{0}-\cdots-a_{n-1}\right)}{\left(c_{0}-a_{0}-\cdots-a_{n-2}\right) a_{n-1}}\right]^{1 / 2}, \mp\left[\frac{ \pm c_{0} a_{n-2}}{\left(c_{0}-a_{0}-\cdots-a_{n-3}\right)\left(c_{0}-a_{0}-\cdots-a_{n-2}\right)}\right]^{1 / 2}, \cdots\right. \\
& \left.\cdots, \mp\left[\frac{ \pm c_{0} a_{2}}{\left(c_{0}-a_{0}-a_{1}\right)\left(c_{0}-a_{0}-a_{1}-a_{2}\right)}\right]^{1 / 2}, \mp\left[\frac{ \pm c_{0} a_{1}}{\left(c_{0}-a_{0}\right)\left(c_{0}-a_{0}-a_{1}\right)}\right]^{1 / 2}, \mp\left[\frac{ \pm a_{0}}{c_{0}-a_{0}}\right]^{1 / 2}\right) \\
& \xi_{n}=\left(\mp\left[\frac{ \pm c_{0} a_{n-1}}{\left(c_{0}-a_{0}-\cdots-a_{n-2}\right) a_{n}}\right]^{1 / 2}, \mp\left[\frac{ \pm c_{0} a_{n-2}}{\left(c_{0}-a_{0}-\cdots-a_{n-3}\right)\left(c_{0}-a_{0}-\cdots-a_{n-2}\right)}\right]^{1 / 2}, \cdots\right. \\
& \left.\cdots, \mp\left[\frac{ \pm c_{0} a_{2}}{\left(c_{0}-a_{0}-a_{1}\right)\left(c_{0}-a_{0}-a_{1}-a_{2}\right)}\right]^{1 / 2}, \mp\left[\frac{ \pm c_{0} a_{1}}{\left(c_{0}-a_{0}\right)\left(c_{0}-a_{0}-a_{1}\right)}\right]^{1 / 2}, \mp\left[\frac{ \pm a_{0}}{c_{0}-a_{0}}\right]^{1 / 2}\right)
\end{aligned}
$$



If

From a particular solution $\xi_{i j}$ of (4) other solutions may be obtained as follows.

$$
S=\left[\begin{array}{cccc}
1 & 0 & \cdots & 0 \\
0 & \sigma_{11} & \cdots & \sigma_{1 n} \\
& & \cdots & \\
0 & \sigma_{n 1} & \cdots & \sigma_{n n}
\end{array}\right]
$$

is a cogredient automorph of $E$, that is if $S E S^{\boldsymbol{T}}=E$, then

$$
\xi_{i j}^{\prime}=\xi_{i 1} \sigma_{1 j}+\cdots+\xi_{i n} \sigma_{i j}
$$

is also a solution. If $Q$ is an arbitrary skew matrix of order $n+1$, with first row and column entirely zero, such that $\operatorname{det}(E+Q)(E-Q) \neq 0$, then

$$
S=(E+Q)^{-1}(E-Q)
$$

is a cogredient automorph of $E$ (see [5], p. 65) of the above form. If $E$ is the identity $S$ is orthogonal. We remark that in this latter case (4) determines the distances $d\left(\xi_{i}, 0\right)$ and $d\left(\xi_{i}, \xi_{j}\right), i, j=0,1, \cdots, n, i \neq j$,

$$
d\left(\xi_{i}, 0\right)=\left[\left(c_{0}-a_{i}\right) / a_{i}\right]^{\frac{1}{2}} \quad d\left(\xi_{i}, \xi_{j}\right)=\left[c_{0}\left(a_{i}+a_{j}\right) / a_{i} a_{j}\right]^{k}
$$

The formulas discussed above are minimal; that is, similar formulas cannot be obtained with fewer points. For if a formula could be obtained with $m+1$ points $\nu_{i}, i=0,1, \cdots, m, m<n$, then equation (2) would still hold, where $C$ is the same as betore and

$$
U=\left[\begin{array}{cccc}
1 & \nu_{01} & \cdots & \nu_{0 n} \\
1 & \nu_{11} & \cdots & \nu_{1 n} \\
& & \cdots & \\
1 & \nu_{m 1} & \cdots & \nu_{m n}
\end{array}\right] \quad A=\left[\begin{array}{cccc}
a_{0} & 0 & \cdots & 0 \\
0 & a_{1} & \cdots & 0 \\
& & \cdots & \\
0 & 0 & \cdots & a_{m}
\end{array}\right]
$$

that is, $U$ is a rectangular matrix. Since $U$ and $A$ have rank at most $m+1$, then $U^{\top} A U$ has rank at most $m+1$ and therefore $\operatorname{det}\left(U^{\top} A U\right)=0$. By assumption $\operatorname{det} C \neq 0$ and thus (2) cannot hold for $m<n$.

3. Formulas of degree 3 for centrally symmetric regions. We assume $R$ to be centrally symmetric with respect to the origin; then if $x$ is in $R,-x$ is also in $R$. Let. us further assume $w(-x)=w(x)$ for $x$ in $R$. Then

$$
\int_{R} x_{i} w(x) d x=\int_{R} x_{i} x_{j} x_{k} w(x) d x=0, \quad i, j, k=1, \cdots, n .
$$

We may obtain an integration formula of degree 3 for $R$ with respect to $w(x)$ with $2 n$ points as follows. Take the points to be $\nu_{i},-\nu_{i}, i=1, \cdots, n$, and take $\nu_{k}$, $-\nu_{k}$ to have common weight $a_{k}$. Any $2 n$ points chosen in this way integrate exactly the monomials $x_{i}, x_{i} x_{j} x_{k}$ with respect to $w(x)$ over $R$. In addition we must solve

$$
\begin{aligned}
& a_{1}+a_{2}+\cdots+a_{n}=\frac{1}{2} c_{0} \\
& a_{1} \nu_{1 j} \nu_{1 k}+a_{2} \nu_{2 j} \nu_{2 k}+\cdots+a_{n} \nu_{n j} \nu_{n k}=\frac{1}{2} c_{j k} \quad j, k=1, \cdots, n .
\end{aligned}
$$


The second of these may be written as the matrix equation (2) where now

$$
U=\left[\begin{array}{cccc}
\nu_{11} & \nu_{12} & \cdots & \nu_{1 n} \\
\nu_{21} & \nu_{22} & \cdots & \nu_{2 n} \\
& \cdots & \\
\nu_{n 1} & \nu_{n 2} & \cdots & \nu_{n n}
\end{array}\right] \quad A=\left[\begin{array}{cccc}
a_{1} & 0 & \cdots & 0 \\
0 & a_{2} & \cdots & 0 \\
0 & 0 & \cdots & a_{n}
\end{array}\right] \quad C=\frac{1}{2}\left[\begin{array}{cccc}
c_{11} & c_{12} & \cdots & c_{1 n} \\
c_{12} & c_{22} & \cdots & c_{2 n} \\
& \cdots & \\
c_{1 n} & c_{2 n} & \cdots & c_{n n}
\end{array}\right]
$$

and where we assume $-\infty<c_{0}<\infty$ and $0<|\operatorname{det} C|<\infty$.

We solve this equation by a method similar to that of the preceding section. We find a non-singular matrix $T$ such that

$$
T^{\top} U^{\top} A U T=T^{\top} C T=E
$$

where $E$ is diagonal with elements \pm 1 . Again it is convenient to assume

$$
E=[1, \cdots, 1,-1, \cdots,-1]
$$

where the first $p$ elements are $+1,0 \leqq p \leqq n$. Now writing

$$
U T=\left[\begin{array}{cccc}
\xi_{11} & \xi_{12} & \cdots & \xi_{1 n} \\
\xi_{21} & \xi_{22} & \cdots & \xi_{2 n} \\
& \cdots & \\
\xi_{n 1} & \xi_{n 2} & \cdots & \xi_{n n}
\end{array}\right]=\left[\begin{array}{cccc}
\nu_{11} & \nu_{12} & \cdots & \nu_{1 n} \\
\nu_{21} & \nu_{22} & \cdots & \nu_{2 n} \\
& \cdots & \\
\nu_{n 1} & \nu_{n 2} & \cdots & \nu_{n n}
\end{array}\right] \quad\left[\begin{array}{cccc}
\tau_{11} & \tau_{12} & \cdots & \tau_{1 n} \\
\tau_{21} & \tau_{22} & \cdots & \tau_{2 n} \\
& \cdots & \\
\tau_{n 1} & \tau_{n 2} & \cdots & \tau_{n n}
\end{array}\right]
$$

the $\xi_{i j}$ may be solved for in terms of the $a_{i}$. This gives

(5) $\xi_{i 1} \xi_{j 1}+\cdots+\xi_{i p} \xi_{j p}-\xi_{i, p+1} \xi_{j, p+1}-\cdots-\xi_{\text {in }} \xi_{j n}=\frac{1}{a_{i}} \delta_{i j} \quad i, j=1, \cdots, n$

precisely $n-p$ of the $a_{i}$ must be negative in order that the $\xi_{i}$ be real. (5) is

If $a_{1}, \cdots, a_{p}$ are positive and $a_{p+1}, \cdots, a_{n}$ negative a particular solution of

$$
\xi_{i}=\left(0, \cdots, 0, \sqrt{1 /\left|a_{i}\right|}, 0, \cdots, 0\right) \quad i=1, \cdots, n
$$

where the $i$ th component of $\xi_{i}$ is non-zero. If $S=\left(\sigma_{i j}\right)$ is any cogredient automorph of $E$ then $\xi_{i j}^{\prime}=\xi_{i 1} \sigma_{1 j}+\cdots+\xi_{i n} \sigma_{n j}$ is also a solution of $(\overline{5})$. If $E$ is the identity, that is, $C$ is positive-definite, the solutions of (5) correspond to the sets of $n$ orthogonal vectors in the space having the property that the $i$ th vector of each set is a distance $\sqrt{1 / a_{i}}$ from the origin.

4. Concluding remarks. The importance of the result given in this paper for formulas of degree 2 is that it is the first result (other than the trivial one point formula, the centroid of $R$, which integrates any linear function) which holds for an arbitrary region in $n$-dimensional space and which gives all such formulas containing the minimum number of points.

A question, which may have some practical importance, which may be asked about the above formulas of degree 2 concerns the conditions $R$ must satisfy, say for $w(x) \equiv 1$, in order that such a formula will exist with all of its points interior to $R$. For example, can a formula interior to $R$ be found if $R$ is convex? if $R$ is star-like about its centroid?

The error bound of von Mises [6] for $n$-dimensional integration formulas is very well suited for use with the formulas developed in this paper. In a later paper we will give specific values of this error bound for various known formulas. 
I am especially indebted to Dr. P. C. Hammer for many discussions concerning this subject.

University of Wisconsin

Madison, Wisconsin

1. G. Grongiev, "Formulas of mechanical quadrature with minimal numbers of terms for multiple integrals," Doklady Akad. Nauk. SSSR, v. 83, 1952, p. 521-524. (Russian)

2. G. GEORGIEV; "Formulas of mechanical quadrature with equal coefficients for multiple integrals," Doklady Akad. Nauk SSSR, v. 89, 1953, p. 389-392. (Russian)

3. G. Georgiev, "Formulas of mechanical cubature with minimal numbers of terms," Rozprawy Matematyczne, No. 8, 1955, p. 72. (Russian; English summary).

4. P. C. HAMMER \& A. H. STROUD, "Numerical evaluation of multiple integrals II," MTAC, v. 12,1958, p. $272-280$.

5. C. C. MACDU FFeE, Theory of Matrices, Chelsea, New York, 1946.

6. R. voN MisEs, "Numerische Berechnung mehrdimensionaler Integrale," Zeit. angew. Math. Mech., v. 34, 1954, p. 201-210.

7. H. C. ThACHER, JR., "Optimum quadrature formulas in s dimensions," MTAC, v. 11, 1957, p. 189-194. 